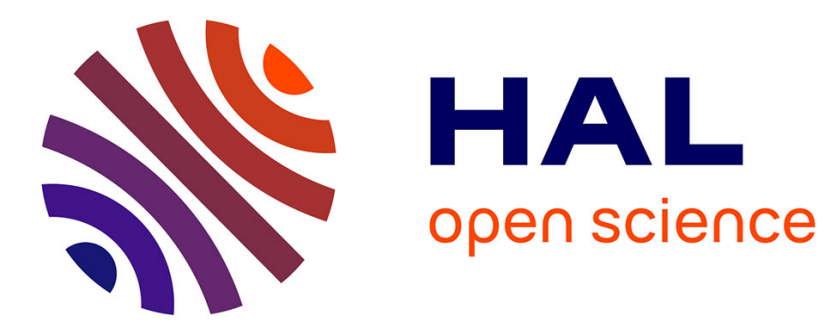

\title{
Injection time controls the final morphology of nanocrystals during in situ-seeding synthesis of silver nanodisks
}

Cecilia Gestraud, Pierre Roblin, Jeffrey F. Morris, Martine Meireles, Yannick Hallez

\section{To cite this version:}

Cecilia Gestraud, Pierre Roblin, Jeffrey F. Morris, Martine Meireles, Yannick Hallez. Injection time controls the final morphology of nanocrystals during in situ-seeding synthesis of silver nanodisks. CrystEngComm, 2020, 22 (10), pp.1769-1778. 10.1039/c9ce01854a . hal-02869704

\section{HAL Id: hal-02869704 https://hal.science/hal-02869704}

Submitted on 16 Jun 2020

HAL is a multi-disciplinary open access archive for the deposit and dissemination of scientific research documents, whether they are published or not. The documents may come from teaching and research institutions in France or abroad, or from public or private research centers.
L'archive ouverte pluridisciplinaire HAL, est destinée au dépôt et à la diffusion de documents scientifiques de niveau recherche, publiés ou non, émanant des établissements d'enseignement et de recherche français ou étrangers, des laboratoires publics ou privés. 


\section{OATAO \\ Open Archive Toulouse Archive Ouverte}

\section{Open Archive Toulouse Archive Ouverte (OATAO)}

OATAO is an open access repository that collects the work of Toulouse researchers and makes it freely available over the web where possible

This is an author's version published in: $\underline{\text { http://oatao.univ-toulouse.fr/26111 }}$

Official URL: https://doi.org/10.1039/c9ce01854a

\section{To cite this version:}

Gestraud, Cecilia $\rightleftharpoons$ and Roblin, Pierre Martine $\rightleftharpoons$ and Hallez, Yannick $\stackrel{5}{-}$ Injection time controls the final morphology of nanocrystals during in situ-seeding synthesis of silver nanodisks. (2020) CrystEngComm, 22 (10). 1769-1778. ISSN 1466-8033

Any correspondence concerning this service should be sent to the repository administrator: tech-oatao@listes-diff.inp-toulouse.fr 


\title{
Injection time controls the final morphology of nanocrystals during in situ-seeding synthesis of silver nanodisks $\dagger$
}

\author{
Cécilia Gestraud, ${ }^{\text {ab }}$ Pierre Roblin, ${ }^{a}$ Jeffrey F. Morris, (D) ${ }^{c}$ \\ Martine Meireles iD *a and Yannick Hallez iD *a
}

\begin{abstract}
We investigate an in situ-seeding synthesis of silver nanodisks based on the sequential addition of weak and strong reducing agents, ascorbic acid and sodium borohydride respectively, to silver nitrate at room temperature and in the presence of polyvinylpyrrolidone. Well-defined silver nanodisks with a uniform thickness of $8 \mathrm{~nm}$ and a distribution of diameters around a mean of $22 \mathrm{~nm}$ can thus be prepared, the diameter being tunable. We report and explain the influence of important experimental control parameters on the quality of the nanoparticle dispersion synthesized. In particular, we show that a dispersion containing exclusively nanodisks can only be obtained if the second reducer is injected in a specific time window at room temperature. The rapid injection of concentrated borohydride triggers a burst nucleation creating seeds with structural defects. Seeds are then grown from silver atoms produced by the reduction of remaining silver ions by ascorbic acid. When the rate of production of these atoms is slow enough, seeds grow along the seed defects and only nanodisks are obtained. The ability to maintain a slow enough production rate after burst nucleation is thus essential. This critical reduction rate depends on the injection time as the latter determines the amount of reactants remaining after nucleation. The influence of temperature is subtle as it affects both reaction kinetics and adatom diffusion on the nanoparticle surfaces. Provided these subtle kinetic effects are understood and under control, the present in situ-seeding synthesis yields good quality nanodisk dispersions in only a few minutes. These results suggest realistic and robust pathways for a scalable production of silver nanodisks in continuous flow reactors most suited to industrial needs.
\end{abstract}

Metallic nanoparticles are well known for producing the plasmon resonance phenomenon. The associated strong enhancement of electromagnetic fields near their surface presents many applications in photovoltaics, ${ }^{1-3}$ photocatalysis, ${ }^{4-6}$ biosensing $^{7}$ or medical applications ${ }^{8}$ among others. Controlling the resonance wavelength can be quite important in some applications such as surface-enhanced Raman spectroscopy (SERS). ${ }^{9-11}$ As this wavelength depends on several parameters among which the nanoparticle size and shape play an important role, ${ }^{12,13}$ it is highly desirable to engineer metallic nanoparticles with finely tunable size and shape, and as monodisperse as possible.

The shape of gold nanocrystals can be controlled quite accurately, but it is not the best material for plasmonics. On

\footnotetext{
${ }^{a}$ Laboratoire de Génie Chimique, Université de Toulouse, CNRS, INPT, UPS, Toulouse, France. E-mail: martine.meireles-masbernat@univ-tlse3.fr, yannick.hallez@univ-tlse3.fr

${ }^{b}$ FERMaT, Université de Toulouse, CNRS, INPT, INSA, UPS, Toulouse, France

${ }^{c}$ Benjamin Levich Institute, City College of City University of New York, New York, USA

$\dagger$ Electronic supplementary information (ESI) available. See DOI: 10.1039/ c9ce01854a
}

the other hand, silver naturally exhibits very good plasmonic performance but the shape and size of silver nanoparticles (AgNP) are typically more difficult to control. Plate-like nanoparticles such as silver nanodisks (AgND) are very promising as they feature a sharp plasmon resonance peak located at a wavelength depending on their aspect ratio ${ }^{14}$ together with a high surface-to-volume ratio interesting for surface dependent applications such as, of course, adsorbed molecule detection and identification but also heterogeneous catalysis or anti-microbial activity. ${ }^{15,16}$ There is, therefore, a strong interest for the reliable production of silver nanodisks with well controlled, tunable size and shape.

Producing anisotropic nanoparticles is appealing, but definitely not straightforward as thermodynamics will favor isotropic shapes. ${ }^{17}$ A classical strategy to force anisotropy is to use surfactants either acting as capping agents ${ }^{18}$ or selfassembling in anisotropic micelles serving as templates. ${ }^{19}$ Another strategy to grow silver anisotropic particles is to work far from equilibrium. This promotes the formation of stacking faults in their crystalline structure. The growth of nanocrystals into $2 \mathrm{D}$ particles such as triangular or hexagonal 
prisms is then associated with the presence of one or several twin planes in an otherwise FCC structure. These defects generate reentrant grooves on the sides of nanoparticles, and these concave regions present sites with a higher number of potential neighbors per adatom compared to flat faces and become therefore the most energetically favorable locations for the attachment of new adatoms. The newly attached atoms thus propagate the crystal structure along the twin plane until anisotropic particles are obtained. ${ }^{20}$

The reasons why twin planes appear, and their number, is still unclear. A frequent explanation is the following one. An adatom arriving on a FCC crystal can end up on either a FCC site or a HCP site. If it has time to diffuse on the surface before any other adatom arrives near it, it has more probability to settle in a most energetically favored FCC site. This is the thermodynamic picture. A kinetic regime is obtained when the rate of addition of adatoms is so high that an adatom can arrive on an HCP site just before a second atom reaches it and stabilizes this structure by increasing the coordination of both atoms. ${ }^{17}$ In other words, the characteristic time for adatom addition $\tau_{\mathrm{r}}$ is smaller than the characteristic time for surface diffusion $\tau_{\mathrm{d}}$. Such a competition between addition and surface migration rates has been exploited for example to direct the growth of bimetallic nanostructures into different shapes. ${ }^{21-23}$

Obtaining twinned seeds is the first condition for a highyield production of well controlled silver nanoplatelets, but it is not enough. The second important condition is to have a growth regime able to maintain the crystalline structure initiated in the seeds and responsible for the presence of the reentrant grooves. As the latter are now the most energetically favorable sites, it is desirable to bring adatoms to the surface as slowly as possible in the growth regime so each new adatom has time to diffuse towards the groove and settle there to start a new layer. Mayer et al. called that "controlled living growth" by analogy with the controlled living polymerization reactions. ${ }^{24}$

The two step picture described above (seed synthesis and seed growth) is generally implemented in two truly separate steps because it permits a fine control of the optimal conditions for each task: fast nucleation and slow growth. Moreover, there is usually a significant rest time after the first step and the seeds have to be washed before they can be used. The transfer of such kinds of protocols to industry is thus complicated. A variant of this method emerged recently as a potential strategy to prepare non-spherical nanostructures with a better yield: the 'in situ-seeding' (or 'one-pot') synthesis during which seeds are synthesized and grown in the same reaction system. ${ }^{25-27}$ In such strategies, a strong reducing agent is usually injected into a medium already containing a weak reducing agent. The role of the strong reducing agent is to trigger homogeneous nucleation almost instantaneously while the role of the weak reducing agent is to grow the seeds slowly, in line with the controlled living growth idea. As presented, in situ-seeding seems to be a simple and attractive method for a scalable production of silver nanodisks. In practice, obtaining a robust protocol can be quite challenging since the different, contradictory kinetic regimes optimal for the synthesis of twinned seeds and for controlled living growth, i.e. fast and slow $\mathrm{Ag}^{0}$ production respectively, can only be obtained by optimizing the stoichiometry of the strong and weak reducing agents, the injection time of the former, and sometimes in a counterintuitive way, temperature.

In this work we discuss the in situ-seeding synthesis proposed by Mahmoud to prepare silver nanodisks. ${ }^{14} \mathrm{We}$ show that the delay for the injection of the strong reducing agent has to lie in a certain time window in order to obtain nanodisks with a well controlled morphology and a satisfactory yield of nanodisks compared to that of unwanted, secondary objects. We explain this effect through the link between injection time and reduction rate immediately after injection. We also discuss the apparently counterintuitive influence of temperature on the optimal injection time window. Altogether our results permit establishment of realistic and robust pathways for a scalable in situ-seedingmethod for the preparation of silver nanodisks.

\section{Experimental section}

\subsection{Chemicals}

Silver nitrate $\left(\mathrm{AgNO}_{3}\right.$, purity $\left.>99 \%\right)$, L-ascorbic acid (AA), sodium borohydride $\left(\mathrm{NaBH}_{4}\right)$ and polyvinylpyrrolidone (PVP, $40000 \mathrm{~g} \mathrm{~mol}^{-1}$ ) were purchased from Sigma-Aldrich as powders and used without further treatment. All the solutions were freshly made by dissolution of the powder into Milli-Q water. The $\mathrm{NaBH}_{4}$ aqueous solution was adjusted at $\mathrm{pH}=11$ and placed in an ice-cooled bath to limit its decomposition. ${ }^{28-30}$

\subsection{Preparation of silver nanodisks}

Silver nanodisks were obtained following the simultaneous multiple asymmetric reduction technique (SMART) developed by Mahmoud. ${ }^{14}$ The synthesis parameters were varied around the following ones, hereafter referred to as the "standard" protocol:

1. In a $100 \mathrm{~mL}$ glass beaker, $0.75 \mathrm{~mL}$ of a $11.8 \mathrm{mM} \mathrm{AgNO}_{3}$ solution is added to $50 \mathrm{~mL}$ of a $0.145 \mathrm{mM}$ aqueous solution of PVP. This mixture is stirred with a standard magnetic stirrer rotating at $1000 \mathrm{rpm}$ under a controlled temperature of $25^{\circ} \mathrm{C}$.

2. $0.120 \mathrm{~mL}$ of a $78 \mathrm{mM}$ AA solution is quickly injected all at once into the reaction mixture at time $t=0 \mathrm{~s}$.

3. After a delay time $t_{\mathrm{i}}=15 \mathrm{~s}, 0.06 \mathrm{~mL}$ of a $1.27 \mathrm{mM}$ $\mathrm{NaBH}_{4}$ solution is further injected all at once.

The last step initiates nucleation and the color of the solution quickly changes from yellow to purple and then to blue. The color of the solution varies more slowly during the next 2 hours, changing from dark blue to purple.

In his original work, Mahmoud reported the influence of the relative quantities of $\mathrm{AgNO}_{3}, \mathrm{NaBH}_{4}$, and $\mathrm{AA}$ on the morphology of nanodisks. ${ }^{14}$ Increasing the quantity of AA led 
to a decrease of the nanodisk aspect ratio, and increasing the $\mathrm{NaBH}_{4}$ concentration led to an increase of the number of seeds, and consequently to the production of smaller objects. Our experiments confirmed this behavior (see ESI†े).

In Mahmoud's work, ${ }^{14}$ the time lapse between the two injections of the reducing agents was not specified. However, we will show in this work that the production of nanoobjects can become quite erratic if this time varies by even a few seconds, depending on the temperature of the synthesis.

\subsection{Characterization techniques}

After the synthesis, silver nanoparticles are collected by centrifugation at $25000 \mathrm{rpm}$ (or 54500 normal gravity) during $25 \mathrm{~min}$ at $25{ }^{\circ} \mathrm{C}$, the supernatant is removed and nanoparticles are re-dispersed in an equal volume of Milli-Q water. Samples were characterized using a JEOL JEM-1400 transmission electronic microscopy (TEM) and a JEM-2100 high-resolution TEM (HRTEM) operated at $120 \mathrm{kV}$. TEM images were analyzed with the ImageJ software to obtain statistics on the diameter and thickness of more than 700 AgND. All samples for TEM and HRTEM were prepared by drop casting using $1 \mu \mathrm{L}$ of AgND dispersion. Absorption spectra were obtained on a Perkin-Elmer UV-visible spectrophotometer with $1 \mathrm{~cm}$ quartz cells. Small angle X-ray scattering (SAXS) measurements were obtained on a Xenocs Xeuss 2.0 laboratory beamline equipped with a Dectris Pilatus $2 \mathrm{M}$ detector.

\section{Results}

TEM images of a dispersion prepared according to the standard protocol reveal mostly circular nanodisks, with a few triangular or hexagonal exceptions (Fig. 1, top). Some of the particles stand on their edge, allowing a measure of their thickness. The image analysis yields a very uniform thickness of $h=8 \mathrm{~nm}$ and diameters distributed on a lognormal law with median $d_{\text {med }} \simeq 22 \mathrm{~nm}$ (Fig. 2). These data are in good agreement with the values $h \simeq 7.9 \mathrm{~nm}$ and $d_{\text {med }} \simeq 25.9 \mathrm{~nm}$ reported previously in the same conditions. ${ }^{14}$

The crystalline nature of nanodisks was confirmed using HRTEM (Fig. 1, middle). The regular atomic arrangement on their faces suggests a unique crystal orientation and therefore flat faces. The selected area electron diffraction (SAED) patterns obtained on these nanodisks (Fig. 1, bottom right) are the same as ones observed in similar systems of $\mathrm{Au}$ and $\mathrm{Ag}$ nanoplates by several authors. ${ }^{31-39}$ The bright spots are assigned to $\{220\}$ Bragg reflections (spacing $1.4 \AA$ ) and the weaker spots closer to the center are attributed to $1 / 3\{422\}$ forbidden reflections due to the twin plane defects parallel to the faces of the nanodisks (spacing $2.5 \AA$ ). The Fourier transform of the HRTEM image (Fig. 1, bottom left) confirms the SAED pattern. These patterns confirm that the nanodisks obtained with the present synthesis feature flat top surfaces and one or multiple twin planes parallel to the [111] plane of the FCC crystal structure.

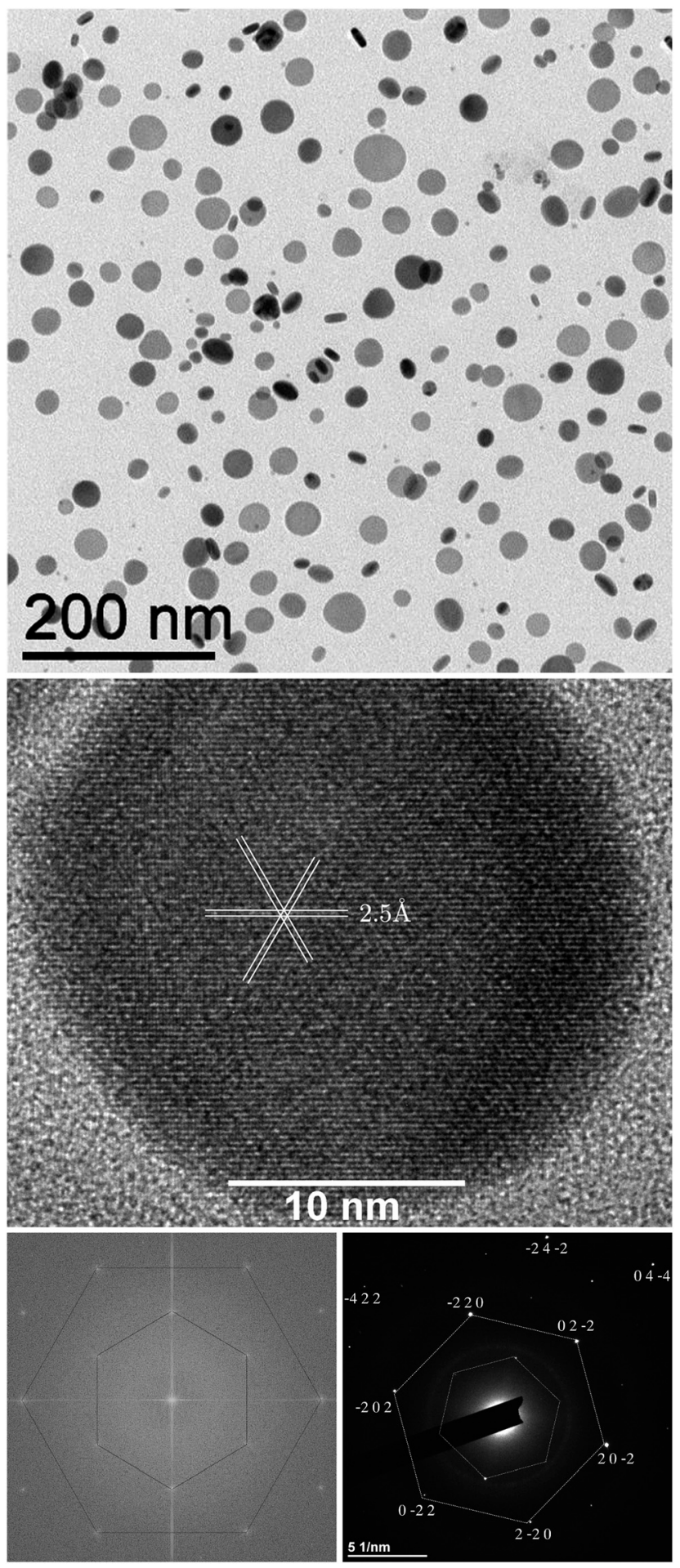

Fig. 1 TEM image of typical dispersion of silver nanodisks (top), HRTEM image of one silver nanodisk standing on its flat face (middle), FFT of the HRTEM image (bottom left) and SAED pattern (bottom right).

TEM has two major drawbacks, namely the limited number of objects that can be observed and the possible modification of the structure of the suspension during sample preparation. As silver nanodisks have plasmonic properties depending on their shape and size, ${ }^{40-43}$ UV-visible 


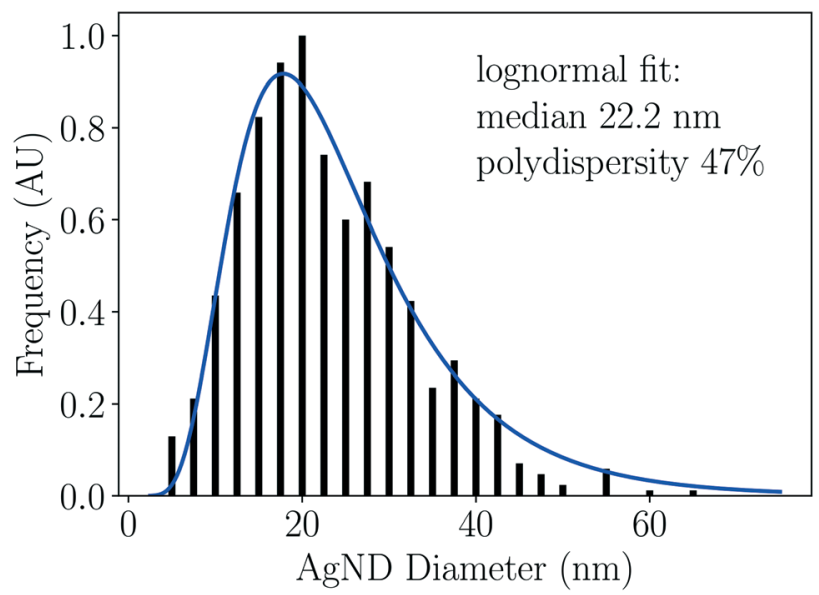

Fig. 2 Silver nanodisk diameter distribution from TEM image analysis.

spectroscopy can also be used to characterize the suspensions in a more statistical way. For plate-like particles, two kinds of plasmon modes exist: the in-plane and out-of-plane modes due to oscillations of electrons along the large and small dimensions of the disks, respectively. Fig. 3 presents an absorption spectrum for a dispersion of silver nanodisks with two main, well resolved peaks plus a less distinct one inbetween. This spectrum is very similar to those observed and calculated by Jin et al. for triangular silver nanoprisms. ${ }^{12}$ The large, low energy peak corresponds to the dipolar in-plane oscillation mode. It is centered around $500 \mathrm{~nm}$ for the standard synthesis conditions but it can be red-shifted with an increase of the AgND aspect ratio. ${ }^{14,36,40,44-47}$ The smaller well defined peak at $340 \mathrm{~nm}$ corresponds to the quadrupolar out-of-plane oscillation mode. To identify the nature of the less distinct observable peak at $410 \mathrm{~nm}$ in the absorption spectrum, we performed discrete dipole approximation (DDA) calculations at fixed ND orientation and light polarization (ESI $\dagger$ ). In the present system this peak is related to the dipolar out-of plane

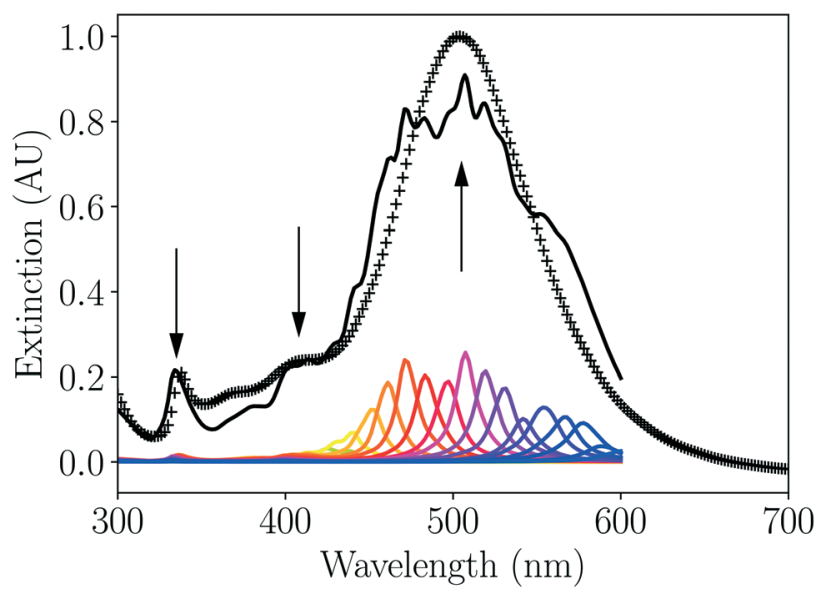

Fig. 3 UV-visible spectrum of a typical dispersion of silver nanodisks as measured experimentally (crosses) and simulated with the DDA (black line, see text for details). The colored spectra were obtained by DDA for monodisperse suspensions of platelets with sizes corresponding to those sampled by the TEM image analysis (Fig. 2). mode, the quadrupolar in-plane mode being very weak. This third peak is however only of limited interest for the characterisation of the prepared dispersions because it is significantly smeared out for ND with arbitrary orientations and polydisperse diameters, and because its position also corresponds to the main dipolar peak of small, residual, 3D objects that are sometimes present. The latter point will be further discussed hereafter.

In order to show the consistency between TEM and UVvisible measurements, a series of discrete dipole approximation calculations have been performed with the DDSCAT software ${ }^{48-50}$ for monodisperse cylinders with diameters between $5 \mathrm{~nm}$ and $47.5 \mathrm{~nm}$ corresponding to the bins of the TEM image analysis of Fig. 2. The resulting extinction spectra are represented in Fig. 3 as colored curves for a cylinder height of $7 \mathrm{~nm}$ and a refractive index $n$ of the aqueous ambient medium of 1.33. A global extinction spectrum including polydispersity effects was then computed by performing a weighted sum of these DDA spectra, the weights being given by the size distribution from the TEM analysis in Fig. 2. The resulting spectra (continuous black line in Fig. 3) is in good agreement with experimental data in terms of peak location and even peak width due to the consideration of polydispersity. Using a nanoplate thickness of $7 \mathrm{~nm}$ in calculations is not precisely consistent with TEM measurements that suggest a value closer to $8 \mathrm{~nm}$. With this latter value, the peaks of the out-of-plane modes are virtually unchanged but the main low-energy peak is blue-shifted by about $15 \mathrm{~nm}$ (not shown). On the other hand, it must be remembered that the AgND are stabilized by a layer of PVP so the refractive index of the ambient medium close to the surface of the nanoparticles might differ from that of water. Using $n=1.54$ corresponding to pure PVP introduces a redshift of the main low-energy peak of about $50 \mathrm{~nm}$ while the two high energy peaks are, again, virtually unchanged. In practice, the effective refractive index close to the surface is between these two values, depending in particular on the grafting density. So it would definitely be possible to use a thickness of $8 \mathrm{~nm}$ in DDA calculations with a tuned value of $n$ to produce a calculated spectra very close to the one represented in Fig. 3. This optimization is however of limited meaning since the PVP grafting density is first unknown and second not the same on all the ND crystalline faces. The main conclusion of this UV-visible response study is the very close agreement with TEM size measurements, to the best of our ability to determine.

Finally, SAXS measurements have been performed to confirm the nanoparticle size and shape in bulk suspension, without the eventual bias introduced by the drying of the sample prior to TEM investigations. The experimental spectrum is reported in Fig. 4 together with a model of polydisperse cylinders with thickness $8 \mathrm{~nm}$ and diameters following the lognormal distribution computed from the TEM measurements. The small discrepancy at the lowest scattering vector $q$ is due to the presence of some small aggregates in the bulk. The almost constant experimental 


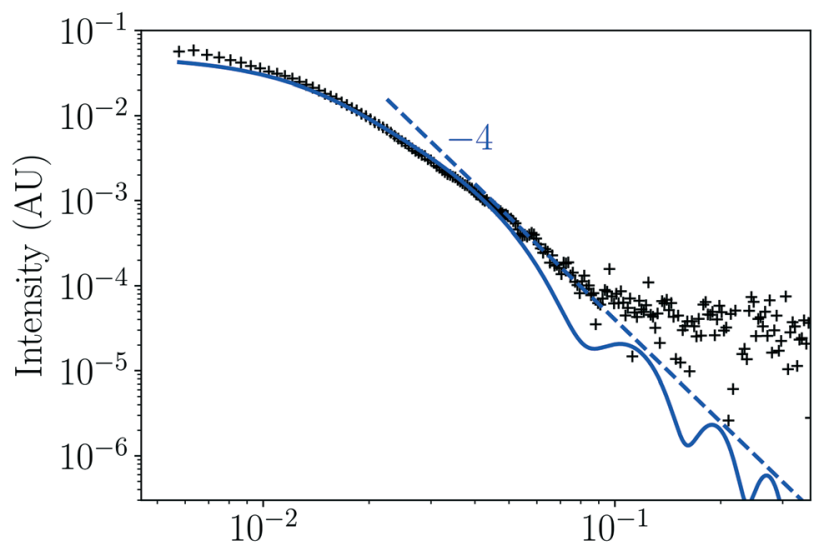

Scattering vector norm $\left(\AA^{-1}\right)$

Fig. 4 SAXS spectrum of an AgND suspension prepared with the standard protocol. Crosses: experimental data; continuous line: theoretical model resulting from the lognormal distribution measured from TEM images.

intensity at the highest $q$ values is due to background noise. The presence of a $q^{-4}$ slope at high $q$ is captured. Obtaining this Porod law shows that the measurements are resolved enough to obtain a signature of the smooth nanoparticle surfaces. ${ }^{51}$ More interestingly, the slope at intermediate $q$ predicted by the model is in nearly perfect agreement with measurements, meaning that the SAXS study confirms a cylindrical particle shape with a distribution of aspect ratios corresponding indeed to the one established by TEM image analysis.

Altogether, TEM images and absorption and SAXS spectra confirm the dispersions prepared in this control experiment contain silver nanodisks with a uniform thickness of $8 \mathrm{~nm}$ and a median diameter of $22 \mathrm{~nm}$.

As mentioned above, the effect of the time lapse between the two injections $t_{\mathrm{i}}$ has not been commented in previous works although in our experiments it turns out to influence the particle shape strongly. In order to show this influence, several dispersions of silver nanoparticles were prepared using the same protocol as the standard one detailed in section 1.2 but with different injection times $t_{\mathrm{i}}$ ranging from 5 to $120 \mathrm{~s}$. The corresponding absorption spectra and TEM images are reported in Fig. 5. Three different behaviors can be identified, with in particular an optimal injection time window.

A "proper" dispersion of nanodisks (i.e. without other $3 \mathrm{D}$ objects) can be obtained at $25^{\circ} \mathrm{C}$ for $t_{\mathrm{i}}$ between 15 and $30 \mathrm{~s}$. As evidenced by UV-visible absorption spectra and TEM images, nanoparticles have a satisfactory disk shape with size and polydispersity similar to those obtained in the standard protocol (Fig. 1 and 3). Moreover, a very good reproducibility can also be obtained in this injection time range.

For an earlier injection of $\mathrm{NaBH}_{4}$ (before $10 \mathrm{~s}$ at $25^{\circ} \mathrm{C}$ ) the presence of different morphologies and sizes of nanoparticles can be observed in TEM images (Fig. 5): nanodisks of equivalent size to those obtained in the standard protocol are present but in addition, a second population of nanoparticles with a size smaller than $10 \mathrm{~nm}$ and therefore probably more isotropic than plate-like. The corresponding UV-visible absorption spectra contain the characteristic peaks of the silver nanodisks described previously, however their relative intensities are different. The peak at $410 \mathrm{~nm}$ presents a higher absorbance than the usual contribution (dipolar outof-plane oscillation). This wavelength actually also corresponds to the plasmonic signature of small spherical, or at least 3D, silver nanoparticles (ESI $\dagger$ ). These results suggest that this range of short injection times is inadequate for all the crystalline defects generated in the seeds to grow in a slow living-growth regime. This hypothesis will be discussed further in the Discussion section.

For injection times $t_{\mathrm{i}}$ larger than about $35 \mathrm{~s}$, TEM images reveal the presence of nanodisks but also smaller and larger 3D particles (Fig. 5). Besides, the absorption spectra are not as peaked as those of successful syntheses, a signature of larger polydispersity, and they are not very reproducible, indicating a poor control of the synthesis.

To understand these results, we propose in the next section a model scenario relying on the concept of living growth for the present in situ-seeding synthesis.

\section{Discussion}

Our results clearly stress the impact of the time interval between the injection of L-ascorbic acid and of $\mathrm{NaBH}_{4}$ on the particle shape and polydispersity. In order to rationalize this effect we propose the following mechanism.

For times $0<t<t_{\mathrm{i}}$, the only reaction is the reduction of silver by ascorbic acid (AA). The reaction rate is ${ }^{52}$

$$
r(t)=k \frac{\left[\mathrm{Ag}^{+}\right][\mathrm{AA}]}{\left[\mathrm{H}^{+}\right]}
$$

where $k$ is a temperature-dependent rate constant. During this first period of time, the concentration of silver atoms increases as depicted on Fig. 6a but not enough to reach the homogeneous nucleation threshold. Metal atoms only accumulate in solution.

At time $t_{\mathrm{i}}$, the strong reducer $\mathrm{NaBH}_{4}$ is injected and is supposed to react quasi-instantaneously compared to the time scale of the synthesis and of the reduction by AA. Note that the minute amount of $\mathrm{NaBH}_{4}$ injected would not be enough to trigger nucleation if it were perfectly and instantaneously mixed. In fact, the reduction of silver by $\mathrm{NaBH}_{4}$ is much faster than the mixing time and nucleation occurs near the surface of the small volume of $\mathrm{NaBH}_{4}$ injected, where the $\mathrm{Ag}^{0}$ concentration can be locally very high before mixing becomes efficient. This nucleation burst is expected to produce seeds with structural defects like twin planes because the kinetics of silver atom production is very fast. $^{17}$

Just after nucleation (time denoted $t_{\mathrm{i}}^{+}$), a large fraction of the silver atoms present before nucleation are consumed and $\left[\mathrm{Ag}^{0}\right]\left(t_{\mathrm{i}}^{+}\right)$is lower than the critical concentration for 

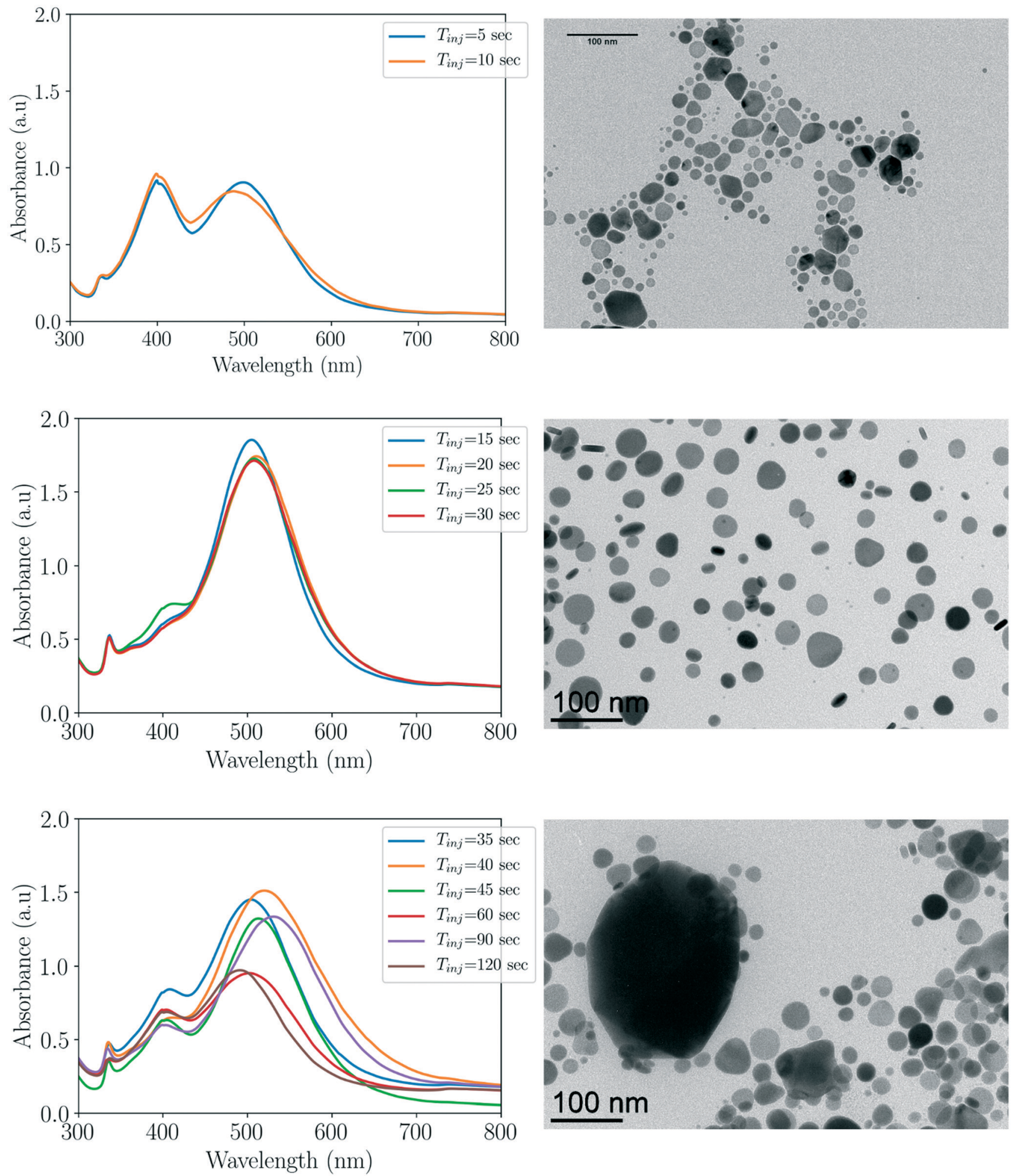

Fig. 5 Influence of the strong reducer injection time $t_{\mathrm{i}}$ on the synthesis illustrated by UV-visible absorption spectra and TEM images.

nucleation. This step is very reminiscent of the hot injection method, ${ }^{53}$ where a fast reaction is rapidly quenched by the temperature shift due to the mixing of reactants. A similar quenching effect exists in the present synthesis as $\mathrm{NaBH}_{4}$, originally prepared at $\mathrm{pH}=11$ and ice-cooled, is quickly decomposed when injected in the $\mathrm{pH} \simeq 3$ solution at room temperature. The analogy with hot injection method stops here, however, because in this method the subsequent growth of seeds is conditioned by a controlled increase of temperature yielding a controlled increase in adatom 
(a)

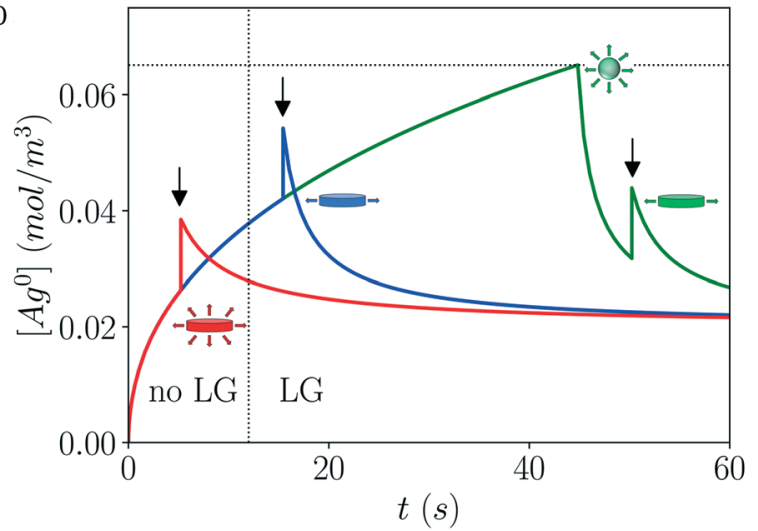

(b)

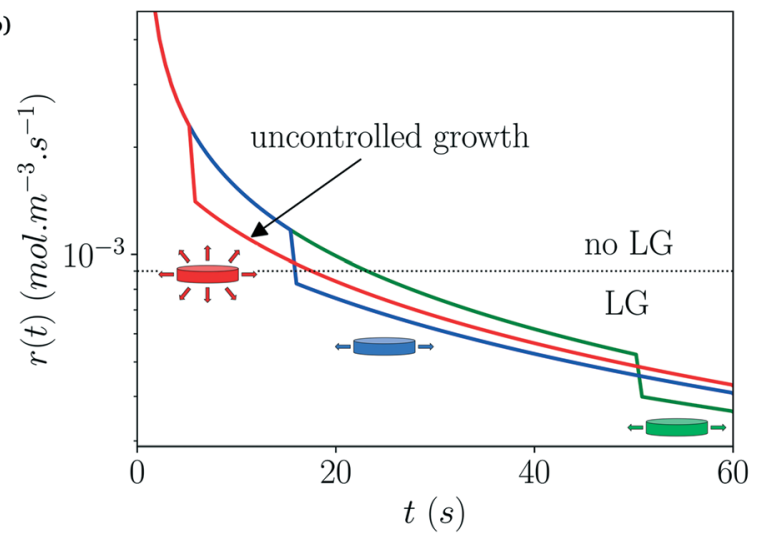

Fig. 6 Schematic representations of the present in situ-seeding synthesis evolution for three different injection times. (a) $\mathrm{Ag}^{\circ}$ concentration evolution. Arrows indicate the strong reducer injection in each case. The horizontal dotted line represents a threshold for spontaneous nucleation. The vertical dotted line indicates the minimum time lag for the injection of the strong reducer required to obtain a living growth regime. (b) Silver reduction rate corresponding to the same scenarios as in Fig. 6a. The dotted line materializes the critical reaction rate $r_{\mathrm{c}}$ below which living growth is obtained.

production rate whereas in the present case the production rate of silver atoms decreases continuously.

As already commented, obtaining twinned seeds is only the first condition to obtain anisotropic and monodisperse nanoparticles. Maintaining a slow atom production rate, say slower than a "critical" value $r_{\mathrm{c}}$, is the second condition to perform growth along the crystal defects in the living growth regime. In the present synthesis, after nucleation, the silver atoms are produced due to the presence of remaining $\mathrm{Ag}^{+}$ and AA. Their production rate at this point is $r\left(t_{\mathrm{i}}^{+}\right)$. It depends in particular on the concentrations of the remaining species right after nucleation $\left[\mathrm{Ag}^{+}\right]\left(t_{\mathrm{i}}^{+}\right)$and $[\mathrm{AA}]\left(t_{\mathrm{i}}^{+}\right)$through eqn (1) and is bound to decrease until the end of the synthesis due to the diminishing concentration of silver ions and/or AA as depicted on Fig. 6b. We can thus see that the ability to maintain a slow enough silver atom production rate depends in particular on the injection time $t_{\mathrm{i}}$, through $\left[\mathrm{Ag}^{+}\right]\left(t_{\mathrm{i}}^{+}\right)$and $[\mathrm{AA}]\left(t_{\mathrm{i}}^{+}\right)$.

Based on this scenario, the influence of the strong reducer injection time can be understood. The concentrations of reduced silver atoms as well as their rate of formation $r(t)$ are represented in Fig. $6 \mathrm{~b}$ for three different injection times 5, 15 and $45 \mathrm{~s}$.

For $t_{\mathrm{i}}=15 \mathrm{~s}$ (blue curve), just after the injection of $\mathrm{NaBH}_{4}$ and the nucleation of seeds, the remaining concentration of AA is low and the production rate of silver atoms $r$ rapidly decreases below the critical value $r_{\mathrm{c}}$ (Fig. 6b). It ensures a controlled living growth regime and a high yield of nanodisks.

For $t_{\mathrm{i}}=5 \mathrm{~s}$ (red curves in Fig. 6), high concentrations of silver ions and AA remain after the nucleation event and the rate of production of silver atoms just after nucleation is still high compared to the critical rate for a controlled living growth. In this regime, seeds are grown too fast with silver atoms deposited both on defects and on crystal faces, leading possibly to non-plate-like objects and to objects with a high polydispersity in size and shape. This corresponds to the syntheses reported in our experiments for $t_{\mathrm{i}}<10 \mathrm{~s}$ in Fig. 5 .

Finally, for $t_{\mathrm{i}}=45 \mathrm{~s}$ (green curves in Fig. 6) the concentration of silver atoms produced by AA before the injection of the second reducer is high enough to induce a first nucleation event before the injection of $\mathrm{NaBH}_{4}$ triggers a second nucleation. The first nucleation event takes place with a moderate $\mathrm{Ag}^{0}$ production rate so the seeds are thought to be produced close to the thermodynamic regime, essentially without defects. The subsequent growth stage then produces large, isotropic 3D objects. The secondary nucleation triggered by the $\mathrm{NaBH}_{4}$ injection is still very fast and generates seeds with defects. These seeds are grown very slowly as the production rate of silver atoms has significantly dropped by this time. Therefore, they turn into proper nanodisks. A characteristic of this last regime is thus to produce both isotropic and plate-like objects in a quite uncontrolled way, in line with the observations reported in Fig. 5 for injection times larger than about $35 \mathrm{~s}$.

To summarize briefly, in a fully thermodynamic synthesis regime where all atoms reach the lowest energy sites both during seed formation and particle growth, only 3D objects can be obtained. A kinetic regime where some atoms end up on "wrong" sites and generate some crystal defects in the seeds can be obtained using a strong reducer such as $\mathrm{NaBH}_{4}$ for nucleation. Once these defects are formed, their edges become the new thermodynamically favored sites. So in order to propagate them to produce anisotropic nanoparticles, the synthesis needs to fall back into a thermodynamic regime immediately after nucleation, during the growth stage. These somewhat contradictory requirements of fast nucleation and slow growth can be met easily in two-step syntheses by adjusting reactant concentrations or temperature separately. In the case of in situ-seeding syntheses, these requirements are met by choosing two reducers with very different kinetics and by adjusting carefully the injection time as described in this work.

As an in situ-seeding synthesis relies on a subtle balance of kinetic effects, its success is likely to be quite dependent on temperature. Unfortunately here the same, unique temperature affects both seed formation and particle growth 
so surprising effects can be observed. When performing the present synthesis at $10{ }^{\circ} \mathrm{C}$, all other things being kept identical to the standard protocol, we obtained results similar to those of the standard synthesis at $25{ }^{\circ} \mathrm{C}$ but the optimal injection time window was much narrower and centered around $10 \mathrm{~s}$ (absorption spectra and a TEM image are reported in ESI $\dagger$ ). At $40{ }^{\circ} \mathrm{C}$, we could obtain very nice nanodisk dispersions for all the injection times tested and ranging from 5 to $120 \mathrm{~s}$ (see ESI $\dagger$ ), indicating a very broad optimal injection time window. One would naively expect that an increase of temperature would be associated to a faster reduction reaction, and consequently to smaller amounts of reactants remaining after injection, to smaller reaction rates during the growth stage, and so to the appearance of a living growth regime. This is actually true at the highest temperatures but not in general. Indeed, the rate constant $k$ is increasing with temperature, but as a consequence the amount of reactants remaining at some prescribed injection time is a decreasing function of temperature. The product can thus be non-monotonic.

Consider for example the results of syntheses conducted at different temperatures but with a fixed injection time $t_{\mathrm{i}}=$ 10 s. At $25{ }^{\circ} \mathrm{C}$ the synthesis is of bad quality (see Fig. 5) whereas it is of excellent quality both at $10{ }^{\circ} \mathrm{C}$ and at $40{ }^{\circ} \mathrm{C}$ (see ESI $†$ ). So the criterion for obtaining living growth would not be a monotonic function of temperature. To verify this hypothesis, we solved eqn (1) numerically until $\mathrm{NaBH}_{4}$ injection and for different temperatures $T$ using an Arrhenius law for $k$. At the end of each simulation and after $\mathrm{NaBH}_{4}$ injection, the reaction rate was computed. We observed that this rate could indeed be a non-monotonic function of temperature, depending on the energy of activation $E_{\mathrm{a}}$. From the reaction rate thus computed after nucleation, one can define an atom production time scale $\tau_{\mathrm{r}}=1 / r\left(t_{\mathrm{i}}^{+}\right)$depending non-monotonically on temperature.

It is then important to remember that temperature has also an influence on the diffusion rate of atoms on surfaces. The diffusion time scale on the surface of a colloid scales as the inverse of a diffusion coefficient, the latter being proportional to the thermal energy $k_{\mathrm{B}} T$. So the diffusion time scale $\tau_{\mathrm{d}}$ is assumed to be $\propto 1 / T$.

Obtaining living growth is possible if the arrival rate of adatoms on a nanoparticle surface is small enough to leave them time to explore the surface. More quantitatively, this corresponds to the condition $\tau_{\mathrm{d}} / \tau_{\mathrm{r}} \ll 1$. The ratio $1 /\left(\operatorname{Tr}\left(t_{\mathrm{i}}^{+}\right)\right) \propto$ $\tau_{\mathrm{d}} / \tau_{\mathrm{r}}$ is reported in Fig. 7 for an injection time of $10 \mathrm{~s}$ and $E_{\mathrm{a}}$ $=37.66 \mathrm{~kJ} \mathrm{~mol}^{-1}$. The non-monotonicity of this ratio and the fact that it reaches a maximum at intermediate temperatures are completely consistent with the experimental observations: at fixed injection time, and for intermediate temperatures, the diffusion time scale is too large compared to the atom production time scale and living growth cannot be obtained. Note that the influence of $\tau_{\mathrm{d}}$ on the ratio $\tau_{\mathrm{d}} \tau_{\mathrm{r}}$ is quite weak compared to the effect of $\tau_{\mathrm{r}}$.

Using the energy of activation $E_{\mathrm{a}}=44.35 \mathrm{~kJ} \mathrm{~mol}^{-1}$ reported in ref. 52 , the ratio $\tau_{\mathrm{d}} \tau_{\mathrm{r}}$ is monotonically increasing with $T$ on

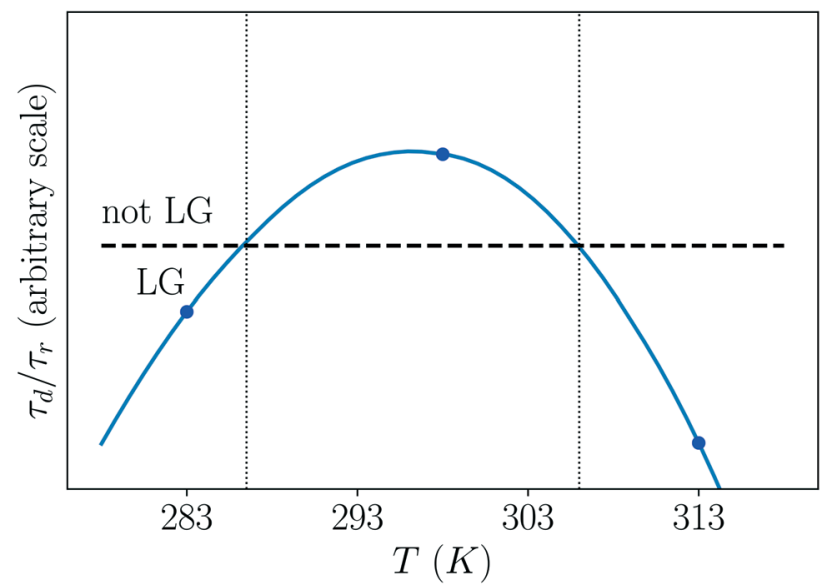

Fig. 7 Temperature dependence of the ratio of the diffusion time scale to the reaction time scale. Simulation conducted with an injection time of $10 \mathrm{~s}$ and with an energy of activation $E_{a}=37.66 \mathrm{~kJ}$ $\mathrm{mol}^{-1}$. The dots correspond to the three temperatures investigated experimentally.

the present temperature range. The value of $E_{\mathrm{a}}$ used here corresponds to a $15 \%$ reduction of this original value. Note that the measurement of the value of $E_{\mathrm{a}}$ reported in ref. 52 was undertaken in very acidic conditions close to $\mathrm{pH} 1$, and that it was done with only ascorbic acid and silver ions. In the present experiments, the $\mathrm{pH}$ varies between 7 and 3 during the synthesis and PVP is present and known to be able to form complexes with silver, so a $15 \%$ uncertainty on $E_{\mathrm{a}}$ does not seem unreasonable. The numerical results reported here highlight the fact that obtaining the required living growth regime by varying temperature can be quite subtle in the present in situ-seeding synthesis.

Note that a faster reduction by ascorbic acid at high temperature should lead to an earlier nucleation without $\mathrm{NaBH}_{4}$ according to the mechanisms described previously in Fig. 6a (imagine the green curve with a higher slope). In fact, the opposite is observed in the experiment realized at $40{ }^{\circ} \mathrm{C}$. This can be explained by an increase of the nucleation threshold with temperature (see ESI $\dagger$ for a qualitative proof).

Finally, note that the results obtained here at $40{ }^{\circ} \mathrm{C}$ are of particular interest for a transfer to industry considering the high yield of nanodisks, the rather large window of possible injection times and the robustness of the synthesis. These properties make the scale up of the protocol easier and more robust.

\section{Conclusions}

The in situ-seeding synthesis described in this study allows silver nanodisks to be obtained successfully and more quickly than with conventional seed and growth methods (only a few minutes instead of several hours). The time interval between the injection of the weak and strong reducing agents was found to be an essential parameter for the control of the final shape and polydispersity of the nanoparticles.

In this study we show that there is an optimal window for this time interval. Injecting the strong reducer too early 
triggers nucleation in a medium still containing a significant amount of silver ions and weak reducer. The production rate of silver atoms after nucleation is too high to propagate these defects properly, in the living growth regime. Injecting the strong reducer too late can leave a chance for a first homogeneous nucleation triggered by the weak reducer action alone, with unwanted isotropic seeds, before the strong reducer injection produces other anisotropic seeds. The dispersions obtained in these conditions are thus quite polydisperse in size and in shape.

The optimal time interval for injection of the two reducers depends significantly and non-monotonically on temperature due to its influence on reaction rate and, to a smaller extent, on the diffusion rate of adatoms on the surface of nanoparticles.

The present observations and conclusions are all consistent with the two following conditions for anisotropic nanoparticle synthesis: a fast nucleation to produce seeds with defects like twin planes, and a slow ensuing metal atom production to leave the latter time to settle into the grooves associated to the defects and propagate them. These conditions are obtained easily in two step syntheses, but a careful interplay between weak and strong reduction kinetics, injection time, and temperature has to be reached in in situseeding syntheses.

However, thanks to the very short synthesis times that in situ-seeding can achieve, this method opens the way for the set-up of high quality, anisotropic, noble metal nanoparticle production in continuous flow reactors most suited to industrial needs.

\section{Conflicts of interest}

There are no conflicts to declare.

\section{Acknowledgements}

We acknowledge the Centre de Micro-Caractérisation Raimond Castaing (UMS 3623 Toulouse), and especially Armel Descamps-Mandine, for the TEM and HRTEM characterizations, the CPER IMATECBIO for financial support for the SAXS equipment, and the UNITI program chaires d'attractivité 2015 ANR-11-IDEX-0002-02 for general financial support.

\section{References}

1 H. A. Atwater and A. Polman, in Materials For Sustainable Energy: A Collection of Peer-Reviewed Research and Review Articles from Nature Publishing Group, World Scientific, 2011, pp. 1-11.

2 C. Clavero, Nat. Photonics, 2014, 8, 95.

3 M. Karg, T. A. König, M. Retsch, C. Stelling, P. M. Reichstein, T. Honold, M. Thelakkat and A. Fery, Mater. Today, 2015, 18, 185-205.

4 Z. Liu, W. Hou, P. Pavaskar, M. Aykol and S. B. Cronin, Nano Lett., 2011, 11, 1111-1116.
5 W. Hou and S. B. Cronin, Adv. Funct. Mater., 2013, 23, 1612-1619.

6 S. Sarina, E. R. Waclawik and H. Zhu, Green Chem., 2013, 15, 1814-1833.

7 G. Lu, H. De Keersmaecker, L. Su, B. Kenens, S. Rocha, E. Fron, C. Chen, P. Van Dorpe, H. Mizuno and J. Hofkens, et al., Adv. Mater., 2014, 26, 5124-5128.

8 M.-F. Tsai, S.-H. G. Chang, F.-Y. Cheng, V. Shanmugam, Y.-S. Cheng, C.-H. Su and C.-S. Yeh, ACS Nano, 2013, 7, 5330-5342.

9 M. Moskovits, Rev. Mod. Phys., 1985, 57, 783-826.

10 S. Nie and S. R. Emory, Science, 1997, 275, 1102-1106.

11 E. Le Ru and P. Etchegoin, Principles of Surface-Enhanced Raman Spectroscopy, 1st edn, 2006.

12 R. Jin, Y. Cao, C. A. Mirkin, K. Kelly, G. C. Schatz and J. Zheng, Science, 2001, 294, 1901-1903.

13 J. J. Mock, M. Barbic, D. R. Smith, D. A. Schultz and S. Schultz, J. Chem. Phys., 2002, 116, 6755-6759.

14 M. A. Mahmoud, Cryst. Growth Des., 2015, 15, 4279-4286.

15 S. Prabhu and E. K. Poulose, Int. Nano Lett., 2012, 2, 32.

16 L. Ge, Q. Li, M. Wang, J. Ouyang, X. Li and M. M. Xing, Int. J. Nanomed., 2014, 9, 2399.

17 T. K. Sau and A. L. Rogach, Adv. Mater., 2010, 22, 1781-1804.

18 Y. Zhou, S. H. Yu, C. Y. Wang, X. G. Li, Y. R. Zhu and Z. Y. Chen, Adv. Mater., 1999, 11, 850-852.

19 N. R. Jana, L. Gearheart and C. J. Murphy, Chem. Commun., 2001, 617-618.

20 C. Lofton and W. Sigmund, Adv. Funct. Mater., 2005, 15, 1197-1208.

21 Y. Yang, W. Wang, X. Li, W. Chen, N. Fan, C. Zou, X. Chen, X. Xu, L. Zhang and S. Huang, Chem. Mater., 2012, 25, 34-41.

22 J. Zeng, C. Zhu, J. Tao, M. Jin, H. Zhang, Z.-Y. Li, Y. Zhu and Y. Xia, Angew. Chem., Int. Ed., 2012, 51, 2354-2358.

23 C. Zhu, J. Zeng, J. Tao, M. C. Johnson, I. Schmidt-Krey, L. Blubaugh, Y. Zhu, Z. Gu and Y. Xia, J. Am. Chem. Soc., 2012, 134, 15822-15831.

24 M. Mayer, L. Scarabelli, K. March, T. Altantzis, M. Tebbe, M. Kociak, S. Bals, F. J. García de Abajo, A. Fery and L. M. LizMarzÃą, Nano Lett., 2015, 15, 5427-5437.

25 T. K. Sau and C. J. Murphy, Philos. Mag., 2007, 87, 2143-2158.

26 X. Jiang, Q. Zeng and A. Yu, Nanotechnology, 2006, 17, 4929.

27 C.-T. Lee, H. Wang, M. Zhao, T.-H. Yang, M. Vara and Y. Xia, Chem. - Eur. J., 2019, 25, 5322-5329.

28 J. Polte, X. Tuaev, M. Wuithschick, A. Fischer, A. F. Thuenemann, K. Rademann, R. Kraehnert and F. Emmerling, ACS Nano, 2012, 6, 5791-5802.

29 J. Andrieux, Degradation of $\mathrm{NaBH}_{4}, 2009$.

30 A.-J. Hung, S.-F. Tsai, Y.-Y. Hsu, J.-R. Ku, Y.-H. Chen and C.-C. Yu, Int. J. Hydrogen Energy, 2008, 33, 6205-6215.

31 V. Germain, J. Li, D. Ingert, Z. L. Wang and M. P. Pileni, J. Phys. Chem. B, 2003, 107, 8717-8720.

32 A. I. Kirkland, D. A. Jefferson, D. G. Duff, P. P. Edwards, I. Gameson, B. F. G. Johnson and D. J. Smith, Proc. R. Soc. London, Ser. A, 1993, 440, 589-609. 
33 C. Lofton and W. Sigmund, Adv. Funct. Mater., 2005, 15, 1197-1208.

34 T. C. R. Rocha and D. Zanchet, J. Phys. Chem. C, 2007, 111, 6989-6993.

35 J. Zeng, J. Tao, W. Li, J. Grant, P. Wang, Y. Zhu and Y. Xia, Chem. - Asian J., 2011, 6, 376-379.

36 C. S. Ah, Y. J. Yun, H. J. Park, W.-J. Kim, D. H. Ha and W. S. Yun, Chem. Mater., 2005, 17, 5558-5561.

37 Z. Yi, X. Xu, X. Wu, C. Chen, X. Li, B. Luo, J. Luo, X. Jiang, W. Wu, Y. Yi and Y. Tang, Appl. Phys. A: Mater. Sci. Process., 2013, 110, 335-342.

38 D. M. Ledwith, A. M. Whelan and J. M. Kelly, J. Mater. Chem., 2007, 17, 2459.

39 D. Aherne, D. M. Ledwith, M. Gara and J. M. Kelly, Adv. Funct. Mater., 2008, 18, 2005-2016.

40 K. L. Kelly, E. Coronado, L. L. Zhao and G. C. Schatz, J. Phys. Chem. B, 2003, 107, 668-677.

41 B. J. Wiley, S. H. Im, Z.-Y. Li, J. McLellan, A. Siekkinen and Y. Xia, J. Phys. Chem. B, 2006, 110, 15666-15675.

42 T. K. Sau, A. L. Rogach, F. JÃd'ckel, T. A. Klar and J. Feldmann, Adv. Mater., 2010, 22, 1805-1825.
43 A. Zhang, D.-J. Qian and M. Chen, Eur. Phys. J. D, 2013, 67, 231.

44 E. Stefan Kooij and B. Poelsema, Phys. Chem. Chem. Phys., 2006, 8, 3349.

45 A. Brioude and M. P. Pileni, J. Phys. Chem. B, 2005, 109, 23371-23377.

46 C. Xue and C. A. Mirkin, Angew. Chem., Int. Ed., 2007, 46, 2036-2038.

47 S. Wang, W. Xi, F. Cai, X. Zhao, Z. Xu, J. Qian and S. He, Theranostics, 2015, 5, 251-266.

48 B. T. Draine and P. J. Flatau, J. Opt. Soc. Am. A, 1994, 11, 1491-1499.

49 B. T. Draine and P. J. Flatau, J. Opt. Soc. Am. A, 2008, 25, 2693-2703.

50 P. Flatau and B. Draine, Opt. Express, 2012, 20, 1247-1252.

51 G. Porod, Kolloid-Z., 1951, 124, 83-114.

52 S. P. Mushran, M. C. Agrawal, R. M. Mehrotra and R. Sanehi, J. Chem. Soc., Dalton Trans., 1974, 1460-1462.

53 C. de Mello Donegá, P. Liljeroth and D. Vanmaekelbergh, Small, 2005, 1, 1152-1162. 


\section{Supplementary information}

\section{Influence of the relative quantities of $\mathrm{AgNO}_{3}, \mathrm{NaBH}_{4}$, and $\mathrm{AA}$ on the morphology of nanodisks}

The concentration of AA has been varied, with all the other parameters set to the values of the standard protocol. The UV-visible spectra of the corresponding dispersions are reported in Fig. 1. Increasing the AA concentration decreases the aspect ratio of the nanodisks.

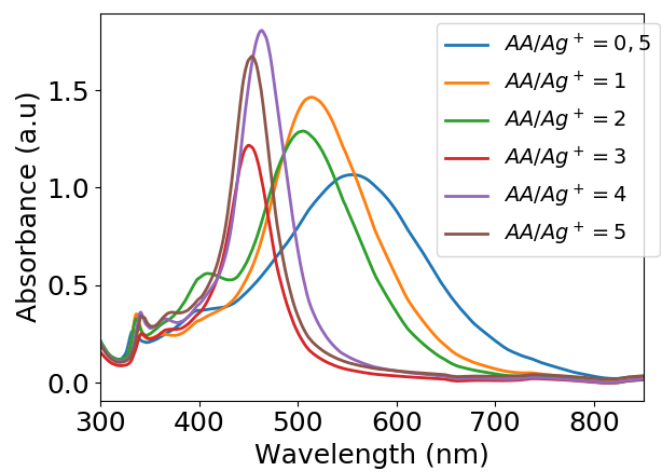

Figure 1: Effect of the variation of the AA concentration on the nanodisk morphology.

The concentration of NaBH4 has also been varied, with all the other parameters set to the values of the standard protocol. The UV-visible spectra of the corresponding dispersions are reported in Fig. 2. Increasing the NaBH4 concentration decreases the size of the nanodisks due to an increase of the number of available seeds.

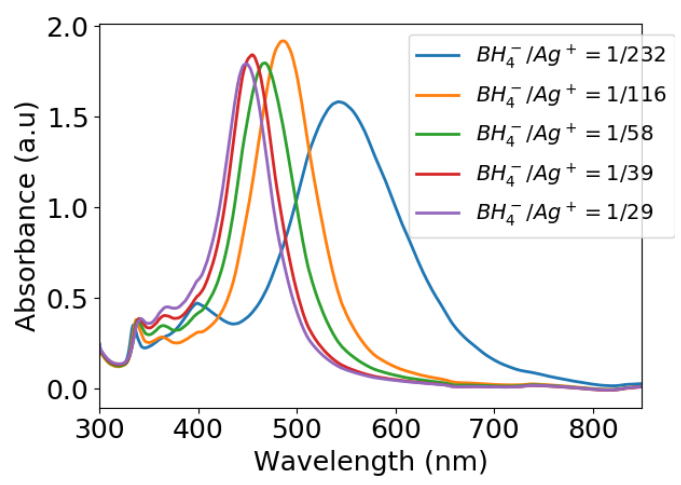

Figure 2: Effect of the variation of the NaBH4 concentration on the nanodisk morphology. 


\section{Syntheses with only one reducing agent}

Syntheses were conducted with only ascorbic acid (weak reducer) or sodium borohydride (strong reducer) to show that using the two reducers simultaneously is necessary for obtaining anisotropic particles. The results of syntheses led with ascorbic acid only or $\mathrm{NaBH}_{4}$ only are presented in Figs. 3 and 4, respectively.

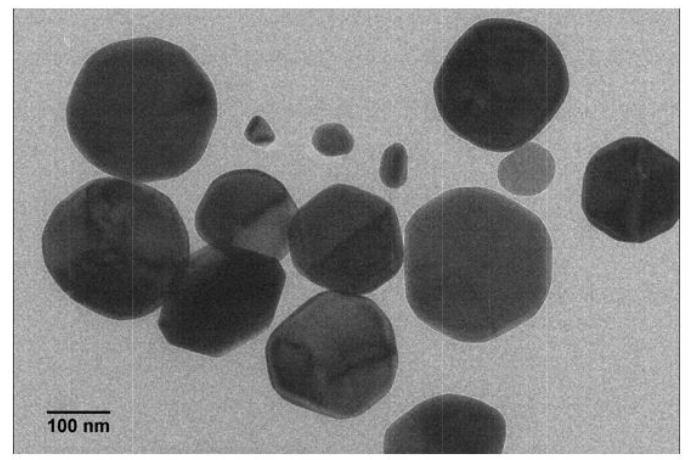

Figure 3: TEM image of silver nanoobjects synthesized using solely L-ascorbic acid and stabilized by PVP, Scale bar: $100 \mathrm{~nm}$. There are no more plasmonic effects with these large particles : the suspension is only turbid.

a)

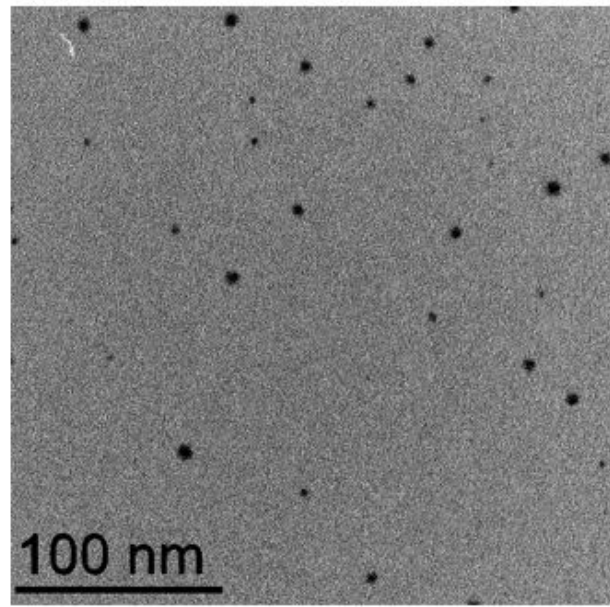

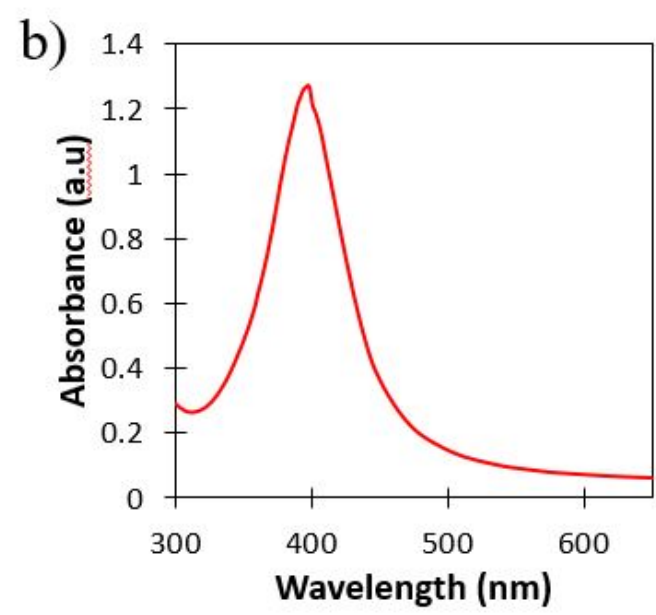

Figure 4: a) TEM image of an example of silver nanoobjects synthesized using solely $\mathrm{NaBH}_{4}$ as reducing agent and stabilized by PVP, Scale bar: $100 \mathrm{~nm}$; b) Corresponding UV-Visible spectra showing the typical band of these 3D silver nanoobjects.

\section{DDA simulation of a single platelet decomposing in-plane and out- of-plane modes}

DDA simulations have been conducted with prescribed light polarization and orientation of a nanodisk in order to isolate the in-plane and out-of-planes plasmon modes. The results reported in Fig. 5 show that the in-plane oscillation is responsible for the main low energy peak only and that the other two smaller peaks correspond dipolar and quadrupolar the out-of-plane modes. 


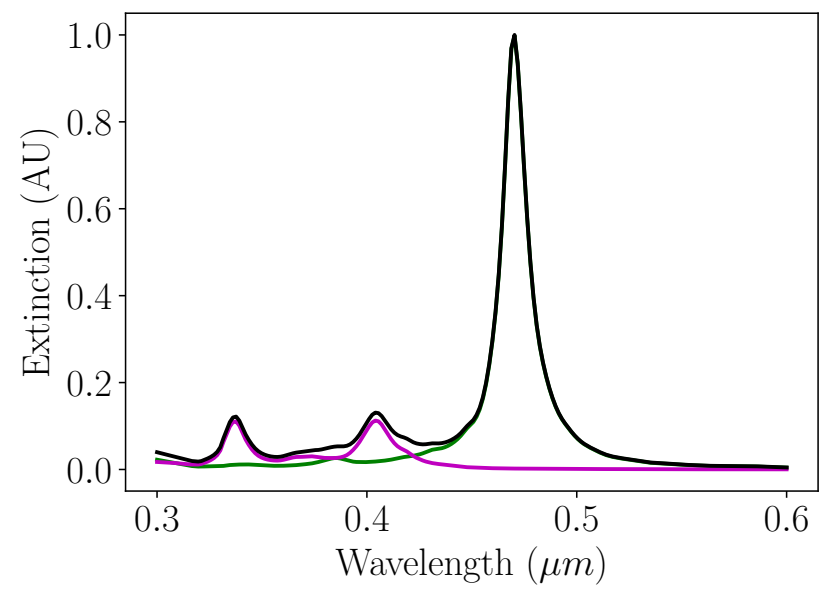

Figure 5: In-plane (green) and out-of-plane (magenta) spectra corresponding to pure in-plane and out-of-plane excitation with prescribed light polarization in DDA simulations. The black curve is the sum. The AgND considered is a cylinder of height $8 \mathrm{~nm}$ and diameter $22 \mathrm{~nm}$.

\section{In-situ-seeding syntheses conducted above or below room tempera- ture.}

The standard synthesis protocol described thoroughly in the article has been reproduced at $40^{\circ} \mathrm{C}$ and $10^{\circ} \mathrm{C}$. The corresponding characterizations of the resulting dispersions for $\mathrm{NaBH}_{4}$ injection times ranging between $5 \mathrm{~s}$ and 120 $\mathrm{s}$ are reported in Figs. 6 and 7.
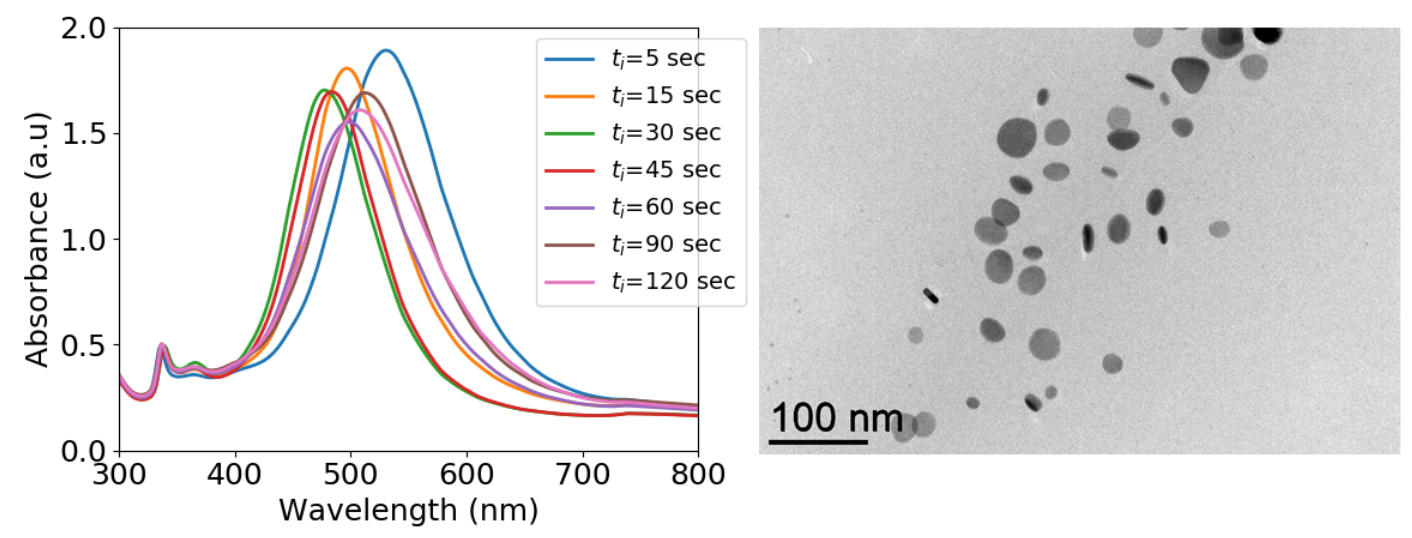

Figure 6: Influence of $t_{i}$ on the synthesis at $40^{\circ} \mathrm{C}$.

\section{Temperature dependence of the nucleation threshold.}

A qualitative evaluation of the temperature dependence of the silver nanocrystals nucleation threshold has been carried out using only silver ions and $\mathrm{NaBH}_{4}$. The silver ion concentration was set to $0.1733 \mathrm{mM}$ and the concentration of reducer was varied. The pictures reported in Fig. 8 show that the nucleation threshold increases significantly in the temperature range investigated in the article. 

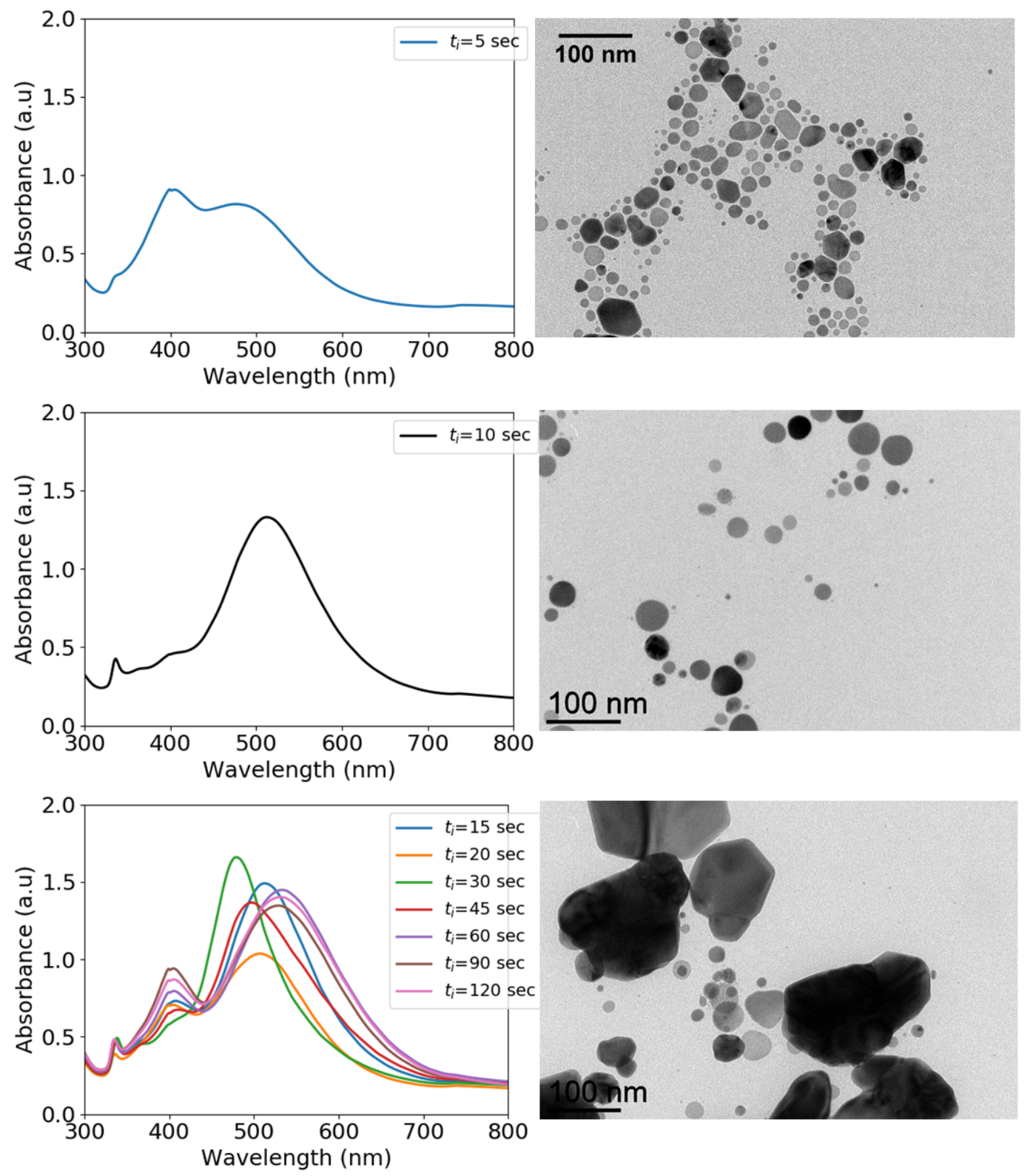

Figure 7: Influence of $t_{i}$ on the synthesis at $10^{\circ} \mathrm{C}$. 


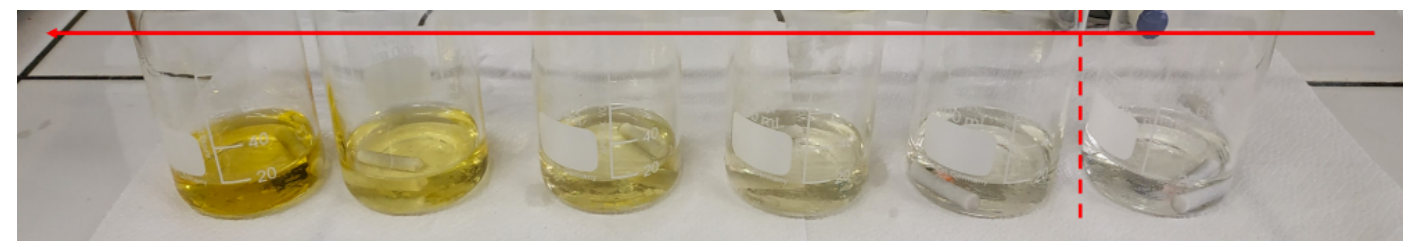

(a) $10^{\circ} \mathrm{C}$

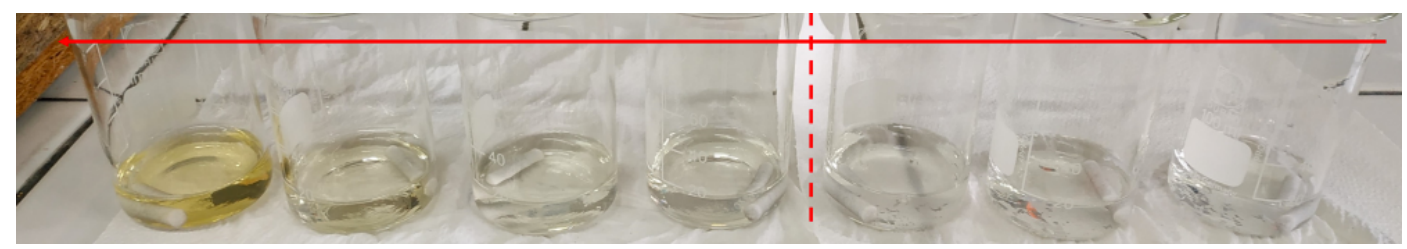

(b) $40^{\circ} \mathrm{C}$

Figure 8: Results of the reduction of silver ions by $\mathrm{NaBH}_{4}$ only for $\mathrm{NaBH}_{4}$ concentrations equal to (from left to right): $1.49 \times 10^{-3}, 7.45 \times 10^{-4}, 3.64 \times 10^{-4}, 1.91 \times 10^{-4}, 9.36 \times 10^{-5}, 4.68 \times 10^{-5}$, and $2.25 \times 10^{-5} \mathrm{mM}$. 